EESTI NSV TEADUSTE AKADEEMIA TOIMETISED. 32. KOIDEE FOOSIKA * MATEMAATIKA. 1983, NR. 2

ИЗВЕСТИЯ АКАДЕМИИ НАУК ЭСТОНСКОИ ССР. ТОМ 32 ФИЗИКА * МАТЕМАТИКА. 1983, № 2

\title{
ON THE ACAUSALITY OF SPIN 3/2 WAVE EQUATIONS
}

\author{
(Presented by H. Keres)
}

The acausality of high-spin wave equations minimally coupled to an external electromagnetic field is connected with the nilpotency of $\beta$-matrices in the free field equation. The acausal propagation is caused by the eigenstates of nilpotent sub-blocks.

\section{Introduction}

The description of high-spin $(s \geqslant 3 / 2)$ particles in interaction with external fields is beset with difficulties. G. Velo and D. Zwanziger $\left.{ }^{1}{ }^{1}\right]$ discovered the acausality at the $c$-number level in the minimally coupled Rarita-Schwinger equation. The acausality means that the wave fronts of classical solutions of wave equations propagate in external fields with speeds greater than that of light. Although the acausality problem has been intensively investigated and various inconsistencies of high-spin equations in external fields have been found, the problem has not yet been solved. It appears that there are no consistent high-spin single particle equations.

In this paper, we show that the acausality phenomenon is closely related to the general algebraic structure of high-spin single particle equations, especially to nilpotency. As it is well known (see, for example, $\left.\left[{ }^{2,3}\right]\right)$, the $\beta$-matrices of high-spin single particle wave equations contain nilpotent sub-matrices. If we decompose the $\beta^{0}$ matrix $\left[{ }^{3}\right] \beta^{0}=$ $=\beta^{\left(s_{1}\right)}+\beta^{\left(s_{2}\right)}+\ldots+\beta^{\left(s_{n}\right)}$, then the matrices $\beta^{(s)}$ of unwanted spins must be nilpotent in the case of more than one spin state. Our statement that the main reason of acausality is the nilpotency, is based on the following facts. 1) From the results of [4], all equations with diagonalizable $\beta$-matrices are causal in the presence of minimal electromagnetic coupling. Diagonalizable matrices have no nilpotent sub-blocks. The $\beta$-matrices of acausal equations are nondiagonalizable and contain therefore nilpotent sub-blocks. No causal equation with nondiagonalizable $\beta$-matrices has been found, and therefore it seems that all normal equations with nilpotent sub-matrices are acausal in the presence of minimal electromagnetic coupling. The normal equation means that we exclude the barnacled equations. As it is shown in $\left[{ }^{5}\right]$, there are causal barnacled equations with nondiagonalizable $\beta$-matrices. It is easy to verify, that in these equations nilpotency is not essential but is caused by barnacles, and the causal barnacled equations are equivalent to the normal equations with diagonalizable $\beta$-matrices. Recently $W$. Cox $\left[{ }^{6}\right]$ proposed two causal $s=0$ equations with nondiagonalizable $\beta$-matrices. As it is shown in $\left[{ }^{7}\right]$, these equations are the barnacled ones and are equivalent to the KemmerDuffin $s=0$ equation. 2) The shock-wave formalism indicates that the acausal modes of propagation are caused by the states eliminated in the free field case by nilpotency. As mentioned before, some of the spin states are eliminated in the free field case. In external fields, there are both - causal modes of propagation and acausal modes of propagation 
[ ${ }^{1}$ ]. Therefore it is important to clarify if there is any correspondence between the propagation modes and spin states. In the spin $3 / 2$ case we shall show that spin $3 / 2$ states propagate causally, whereas spin $1 / 2$ states eliminated in the free field case, propagate acausally in external fields. 3 ) It was shown in a recent paper $\left[{ }^{8}\right]$, that the equations with nilpotent matrices may be classified as unphysical also in the free field case since the parameters corresponding to nilpotent matrices lie in unphysical region.

\section{The Bhabha-Gupta equation}

In this section we deal with the Bhabha-Gupta equation for spins $3 / 2$ and $1 / 2$. The acausality of Bhabha-Gupta equation was examined in $\left.{ }^{9}\right]$. It was shown that it is possible to avoid acausality in the equation with a proper choice of free parameters, but this choice makes the total charge in the free field theory indefinite. Here we demonstrate that this particular choice of parameters eliminates the nilpotency of $\beta^{0}$ matrix. We shall show in the next section that the total charge is indefinite since the equation describes two spin states $-3 / 2$ and $1 / 2$.

We have chosen the Bhabha-Gupta equation as it contains free parameters and thus gives a whole family of high-spin equations. As a special case, we get the Rarita-Schwinger equation, acausality of which is well known $\left[{ }^{1}\right]$, and the Harish-Chandra equation, acausality of which was studied in [ $\left.{ }^{10}\right]$.

We choose the Bhabha-Gupta Lagrangian density in the form

$$
\begin{aligned}
& L=-\bar{\psi}^{\mu}\left(p_{\gamma}-m\right) \psi_{\mu}+3^{-1} \bar{\psi}^{\mu}\left(p_{\mu}-\gamma_{\mu}(p \gamma)\right) \gamma \psi+\bar{\psi}^{\mu} \gamma_{\mu} p \psi- \\
& -d \bar{\psi}^{\mu}\left(p_{\mu}-\gamma_{\mu}(p \gamma)\right) \varphi-\bar{\varphi}\left(\lambda^{-1} p_{\gamma}-m\right) \varphi-d(a \lambda)^{-1} \bar{\varphi} p \psi .
\end{aligned}
$$

Here $\psi_{\mu}$ is a vector-bispinor; $\varphi$, a bispinor, $\bar{\psi}^{\mu}=\left(\psi^{\mu}\right)^{+} \gamma^{0}, \bar{\varphi}=\varphi^{+} \gamma^{0}$, and $p_{\mu}=i \partial_{\mu}$. The constants $a, \lambda$ and $d$ are real and arbitrary.

From (1) we get the following equations:

$$
\begin{gathered}
(p \gamma-m) \psi_{\mu}-3^{-1}\left(p_{\mu}-\gamma_{\mu}(p \gamma)\right) \gamma \varphi-\gamma_{\mu} p \psi+d\left(p_{\mu}-\gamma_{\mu}(p \gamma)\right) \varphi=0, \\
\left(\lambda^{-1} p_{\gamma}-m\right) \varphi+d(a \lambda)^{-1} p \psi=0 .
\end{gathered}
$$

Our Lagrangian density (1) and equation (2) are different from that of $\left[{ }^{9}\right]$, but it is easy to verify that our equation (2) is equivalent to the Bhabha-Gupta equation given in [ $\left.{ }^{9}\right]$ : Multiplying $(2)$ by $\left(p_{\gamma}\right) \gamma^{\mu}$ and $p^{\mu}$, we, similarly as in $\left[{ }^{9}\right]$, obtain

$$
\begin{aligned}
& p \psi=0, \\
& \gamma \psi=-3 d \lambda \varphi .
\end{aligned}
$$

Substituting (3) back into (2), we get the equivalency with the BhabhaGupta equation given in [ $\left.{ }^{9}\right]$. Our Lagrangian density (1) allows to write $(2)$ in a standard form of a first-order wave equation

$$
\left(p_{\mu} \beta^{\mu}-m\right) \Psi=0,
$$

where

$$
\Psi=\left|\begin{array}{c}
\psi_{\mu} \\
\varphi
\end{array}\right| \text {. }
$$

This form gives us a true charge density and is useful in managing nilpotent states (see Sect. 5).

$\beta^{0}$ matrix of equation (4) has a form 


$$
\beta^{0}=\left|\begin{array}{ccccc}
0 & 0 & 0 & 0 & 0 \\
-\frac{2}{3} \gamma_{1} & \frac{2}{3} \gamma_{0} & \frac{1}{3} \gamma_{0} \gamma_{1} \gamma_{2} & \frac{1}{3} \gamma_{0} \gamma_{1} \gamma_{3} & d \gamma_{0} \gamma_{1} \\
-\frac{2}{3} \gamma_{2} & \frac{1}{3} \gamma_{0} \gamma_{2} \gamma_{1} & \frac{2}{3} \gamma_{0} & \frac{1}{3} \gamma_{0} \gamma_{2} \gamma_{3} & d \gamma_{0} \gamma_{2} \\
-\frac{2}{3} \gamma_{3} & \frac{1}{3} \gamma_{0} \gamma_{3} \gamma_{1} & \frac{1}{3} \gamma_{0} \gamma_{3} \gamma_{2} & \frac{2}{3} \gamma_{0} & d \gamma_{0} \gamma_{3} \\
d(a \lambda)^{-1} & 0 & 0 & 0 & \lambda^{-1} \gamma_{0}
\end{array}\right|
$$

The Bhabha-Gupta equation describes $s=3 / 2$ state and $s=1 / 2$ state with the masses $m$ and $\lambda m$, respectively. In the limit $d \rightarrow 0, \lambda \rightarrow \infty$, we get the equation which is equivalent to the Rarita-Schwinger equation with the single $s=3 / 2$ state.

The causality investigation in minimal electromagnetic coupling $p_{\mu} \rightarrow D_{\mu}=p_{\mu}-e A_{\mu}, A_{\mu}$ being the electromagnetic potential, is performed, employing the shock-wave formalism similarly as in $\left[{ }^{9}\right]$, and gives the same result. The normals of characteristic surfaces $n^{\mu}$ are determined by the following condition:

$$
n^{2}\left\{n^{2}+\left[\frac{2 e}{3 m^{2}}\left(1-\frac{3 d^{2}}{2 a}\right)\right]^{2}(\hat{F} n)^{2}\right\}=0,
$$

where $\hat{F}^{\mu v}=2^{-1} \in^{\mu \nu \rho \sigma} F_{\rho \sigma}$ is a dual electromagnetic tensor.

If $3 d^{2} \neq 2 a$, the expression in brackets admits solutions with $\left(n^{0}\right)^{2}>\mathbf{n}^{2}$ and leads, thus, to acausal propagation. In the limit $d \rightarrow 0$, we get the acausality of Rarita-Schwinger equation. Choosing in (6) $3 d^{2}=2 a$ $(a, d \neq 0)$, it is possible to avoid acausality.

Now let us demonstrate that in the case of causal propagation $\beta^{0}$ is diagonalizable, whereas otherwise $\beta^{0}$ is nondiagonalizable and contains nilpotenet sub-matrices. To show this, we, using (5), calculate the minimal polynomials of $\beta^{0}$ :

C a s e $\lambda \neq 1$. 1) $3 d^{2} \neq 2 a$

$$
\beta^{0^{2}}\left(\beta^{0^{2}}-1\right)\left(\beta^{0^{2}}-\lambda^{-2}\right)=0,
$$

2) $3 d^{2}=2 a$

$$
\beta^{0}\left(\beta^{0^{2}}-1\right)\left(\beta^{0^{2}}-\lambda^{-2}\right)=0 ;
$$

C a s e $\lambda=1$. 1) $3 d^{2} \neq 2 a$

$$
\beta^{0^{2}}\left(\beta^{0^{2}}-1\right)=0
$$

2) $3 d^{2}=2 a$

$$
\beta^{0}\left(\beta^{0^{2}}-1\right)=0 .
$$

It follows from (8) and (10) that in the case of causal propagation $\left(3 d^{2}=2 a\right), \beta^{0}$ is diagonalizable. This, in turn, is in accordance with the result of V. Amar and U. Dozzio $\left[{ }^{4}\right]$ that the equations with diagonalizable $\beta^{0}$ matrix are causal in the presence of external electromagnetic field.

In the case of acausal propagation $\left(3 d^{2} \neq 2 a\right),(7)$ and (9) show that $\beta^{0}$ is nondiagonalizable and contains nilpotent sub-matrices.

To summarize, we have shown that in the case of Bhabha-Gupta equation, the acausal propagation is connected with the nilpotency of $\beta^{0}$ matrix in the free field equation. The propagation is causal if and only if $\beta^{0}$ is diagonalizable. Also, it should be mentioned that the nilpotent part is connected with the $s=1 / 2$ states. Decomposing $\beta^{0}=\beta^{(3 / 2)}+\beta^{(1 / 2)}$, 
it is possible to show that $\beta^{(3 / 2)}$ is diagonalizable and satisfies $\beta^{(3 / 2)}\left(\left(\beta^{(3 / 2)}\right)^{2}-1\right)=0$; so $\beta^{(1 / 2)}$ must be nondiagonalizable. This follows from the fact that the choice $d=0$ gives us, according to (2), two equations - Rarita-Schwinger equation for $\psi_{\mu}$ and Dirac equation for $\varphi$ : In the case of Rarita-Schwinger equation, $\beta^{(3,2)}$ is diagonalizable [ [ $]$. In $(2), \beta^{(3 / 2)}$ remains unchanged because the parameters $a, \lambda$ and $d$ altered only $s=1 / 2$ states.

\section{The definiteness of total charge}

Let us also examine the definiteness of total charge $Q=\int d^{3} x j^{0}$. Using (1), we get for $j^{0}$ the following expression:

$$
\begin{aligned}
& \left.j^{0}=-\overline{\psi^{\mu}} \gamma^{0} \psi_{\mu}+3^{-1} \overline{\left(\psi^{0}\right.}-(\bar{\psi} \gamma) \gamma^{0}\right) \psi \psi+\bar{\psi} \gamma \psi^{0}- \\
& -\overline{d \psi^{0}} \varphi+d(\overline{(\psi} \gamma) \gamma^{0} \varphi-\lambda^{-1} \varphi \gamma^{0} \varphi-d(a \lambda)^{-1} \varphi \psi^{0} .
\end{aligned}
$$

To study the definiteness of total charge, it is enough to consider the rest frame in which $p=0$. Then the equations (3) give

$$
\psi^{0}=0, \quad \overrightarrow{\gamma \psi}=3 d \lambda \varphi .
$$

Using (12) in (11), we obtain

$$
Q=\int d^{3} x\left[\overrightarrow{\psi^{+}} \vec{\psi}-\left(3 d^{2} \lambda^{2}+3 d^{2} \lambda+\lambda^{-1}\right) \varphi^{+} \varphi\right]
$$

In order to determine the definiteness of a charge, it is necessary to calculate the charges of different spin states. The Bhabha-Gupta equation describes one spin $3 / 2$ state with mass $m$ and one spin $1 / 2$ state with mass $\lambda m$. Calculating from (5) the corresponding eigenstates, we obtain from (13)

$$
Q_{3 / 2}=\int d^{3} x \vec{\psi}+\vec{\psi}, \quad Q_{1 / 2}=-\left(3 d^{2} \lambda+\lambda^{-1}\right) \int d^{3} x \varphi^{+} \varphi .
$$

The last expressions show that the definiteness of a charge depends on the choice of the sign of $\lambda$. If $\lambda>0$, the total charge is indefinite, if $\lambda<0$, the total charge is definite.

\section{The Fisk-Tait equation}

C. Fisk and W. Tait proposed in [ [11] an equation to describe spin $3 / 2$ particles, which is free from acausality in the minimal electromagnetic coupling. Due to its causality this equation is intensively investigated. In $\left[{ }^{9}\right]$ it is shown that the total charge of Fisk-Tait equation is indefinite. As has been mentioned above, indefiniteness in general shows the multiparticle content of an equation, whereas causality shows that $\beta^{0}$ is diagonalizable.

The general algebraic structure of the Fisk-Tait equation is investigated in [ $\left.{ }^{12}\right]$. It is shown that the Fisk-Tait equation is a reducible one; it describes two spin $3 / 2$ particles with the same mass $m$, and $\beta^{0}$ satisfies $\beta^{0}\left(\beta^{0^{2}}-1\right)=0$.

Recently G. Labontè $\left[{ }^{13}\right]$ proposed a new equation for an antisymmetric tensor-bispinor to describe spin $3 / 2$ field, and demonstrated its causality and indefiniteness. It is easy to verify that there are no good single-mass equations for an antisymmetric tensor-bispinor. The equations we get are all reducible to the simpler ones, such as the Fisk-Tait equation $\left[{ }^{11,12}\right]$ or the Rarita-Schwinger equation. 


\section{The acausality and nilpotent states}

In previous sections we showed that the acausality is connected with the nilpotency of $\beta$-matrices, and therefore the knowledge of algebraic structure of $\beta$-matrices is important. In the acausal equations there are causal modes of propagation as well as acausal ones. In this section we shall show that acausal modes of propagation are caused by the spin states corresponding to the nilpotent sub-blocks in the free field case.

As it is known [9,14], the existence of acausal modes of propagation can be seen if we use the shock-wave formalism. In this section we demonstrate that the bad characteristics we get from the shock-wave formalism, are connected with the states eliminated by nilpotency.

We deal with the Bhabha-Gupta equation: In the decomposition $\beta^{0}=$ $=\beta^{(3 / 2)}+\beta^{(1 / 2)}, \beta^{(3 / 2)}$ remains the same and contains no nilpotent sub-block, $\beta^{(1 / 2)}$ depends on parameters $a, \lambda$ and $d$, and contains, in general, nilpotent sub-block which eliminates some $s=1 / 2$ states. The acausal propagation is connected with the zero eigenvalues of $\beta^{(1 / 2)}$. To show this, we use the shock-wave method. Introducing in (2) the minimal coupling by the substitution $p_{\mu} \rightarrow D_{\mu}=p_{\mu}-e A_{\mu}$, we denote the magnitudes of the discontinuities across the characteristics by the following -

$$
\begin{gathered}
{\left[D_{\mu} \psi_{v}\right]=n_{\mu} k_{v}, \quad\left[D_{\mu} \varphi\right]=n_{\mu} r,} \\
{\left[\psi_{\mu}\right]=[\varphi]=0,}
\end{gathered}
$$

where $n_{\mu}$ are the components of the normal to the characteristic surface, $k_{\mu}$ is the vector-bispinor and $r$ the spinor. If we take the discontinuities, we get from (2)

$$
\begin{gathered}
(n \gamma) k_{\mu}-3^{-1}\left(n_{\mu}-\gamma_{\mu}(n \gamma)\right) \gamma^{k}-\gamma_{\mu} n k+d\left(n_{\mu}-\gamma_{\mu}(n \gamma)\right) r=0, \\
\left(\lambda^{-1} n \gamma\right) r+d(a \lambda)^{-1} n k=0 .
\end{gathered}
$$

The last equation may be written as

$$
\left(n_{\mu} \beta^{\mu}\right) K=0 \text {, }
$$

where

$$
K=\left|\begin{array}{c}
k_{\mu} \\
r
\end{array}\right|
$$

The equation (17) (or (16)) is one of the starting equations of the shock-wave formalism and leads to (6), showing the existence of acausal modes of propagation. To show acausal propagation, one must prove the existence of time-like $\left(n^{2}>0\right)$ normals that satisfy (17), and constraints following from (2). For time-like normals there exists a system, where $n_{\mu}$ takes the form $n_{\mu}=(1,0,0,0)$. Then, from $(17)$, it follows

$$
\beta^{0} K=0 \text {. }
$$

The expression (19) shows that the time-like normals are connected with the zero eigenvalues of $\beta^{0}$. The solutions of (19), in turn, correspond to the $s=1 / 2$ states which in the free field case are eliminated by the nilpotency.

The last statement is proved as follows. From $D^{\mu} \psi_{\mu}=0$, taking discontinuities, we obtain

$$
n^{\mu} k_{\mu}=0 .
$$

Substituting it back into the second equation (16), we get for time-like $n^{\mu}$

$$
r=0 \text {; }
$$




$$
K=\left|\begin{array}{c}
k_{\mu} \\
0
\end{array}\right|,
$$

and the equation (19) reduces to the eigenvalue problem of RaritaSchwinger equation. In the case of Rarita-Schwinger equation, the matrix $\beta^{(1 / 2)}$ is nilpotent and satisfies $\left.\left(\beta^{(1 / 2)}\right)^{2}=0{ }^{3,8}\right]$. Therefore one of the $s=1 / 2$ states is eliminated by the nilpotency, while the other one corresponds to the zeroth eigenvalue of $\beta^{(1 / 2)}$. Since the $s=3 / 2$ states correspond to nonzero eigenvalues of $\beta^{0}$ (more exactly, $\beta^{(3 / 2)}$ ), the solutions of (19) correspond to the spin $1 / 2$. The existence of time-like normals can be proved on the nonzero solutions of (19) which, as we have shown, correspond to the zeroth eigenvalue of nilpotent matrix $\beta^{(1 / 2)}$. Hence the acausal propagation is connected with the nilpotent $s=1 / 2$ states.

The propagation of spin $3 / 2$ states is causal, since $\beta^{(3 / 2)}$ is not altered by the parameters $a, \lambda$ and $d$, and remains the same in the causal or acausal Bhabha-Gupta equation (and, as a special case, in the RaritaSchwinger equation, too).

In $\left.{ }^{8}\right]$ we investigated all the physically acceptable equations with the representation $R(1)=(1,1 / 2) \oplus(0,1 / 2) \oplus(1 / 2,0) \oplus(1 / 2,1)$, where the $\beta^{(3 / 2)}$ remains the same, but $\beta^{(1 / 2)}$ depends on parameters, and describes different $s=1 / 2$ mass states. It appears that the only acausal equation we get is the Rarita-Schwinger equation. It allows to classify the equations with nilpotent matrices, as unphysical already in the free field case, since the corresponding parameters lie in unphysical region $\left[{ }^{8}\right]$. The causality of some equations with $R(1)$, where in addition to spin $3 / 2$ there is spin $1 / 2$ state, was demonstrated in $\left[{ }^{15}\right]$. As it follows from the results of $\left[{ }^{8}\right]$, these equations have diagonalizable $\beta^{0}$ matrices.

It was demonstrated in a recent paper $\left[{ }^{16}\right]$ that in the quantised Rarita-Schwinger equation the anomalies are also connected with spin $1 / 2$ states. This result is in agreement with the results of the section treating on the classical field case.

\section{Conclusions}

In this paper we have shown that the acausality of high-spin equations is connected with the nilpotency of $\beta$-matrices. More exactly, acausal propagation is caused by the eigenstates corresponding to nilpotent sub-matrices. The equations with nilpotent matrices may be classified as unphysical already in the free field case, since the corresponding parameters lie in the unphysical region. This argumentation allows to classify the equations with nilpotent matrices as unphysical.

If we continue this argumentation, the only physical equations are those with diagonalizable $\beta$-matrices. On the other hand, the high-spin equations with diagonalizable $\beta$-matrices are in general multiparticle equations and therefore the consistent description of high-spin states inevitably introduces the states with lower spins. Again, multiparticle equations are indefinite and need an indefinite metric in quatization.

The fact that the multiparticle equations with diagonalizable $\beta$-matrices have no acausal defects in minimal electromagnetic coupling leads to different interpretations. One of the spread interpretations is the following: the high-spin particles are not elementary but the composite ones (see, for example, $\left[{ }^{10}\right]$ ). This interpretation is based on the ideas of gauge theories, where the elementary constituents of matter are leptons and quarks. We think that more realistic is probably the interpretation according to which all the defects lie in the formalism we used. In our standard description of particles we realise the irreducible representations 
of the Poincaré group $(m, s)$ with the help of reducible representations of the Lorentz group. The latter ones have, in general, more components than it is necessary. The role of a relativistically invariant equation is to introduce mass and spin, and eliminate superfluous components. In the free field case the unwanted components may be eliminated, but in external fields we have no guarantee that these components do not enter into the equation. As we have shown, this indeed takes place. Therefore the role of components which are eliminated in the free field case needs further investigation.

Another hope to avoid acausality and get a consistent description of the high-spin field is offered by supergravitation, where the states with spins 2 and $3 / 2$ enter into the same supermultiplet. In spite of the fact that the pure supergravity $(2,3 / 2)$ is free from acausality $\left[{ }^{17}\right]$, the causality in the presence of matter fields is not known. In the pure supergravity spin $3 / 2$ is coupled with the gravitational field and therefore the gravitational coupling is free from acausality. In supergravity, too, the reducible representations of the Lorentz group are used, and therefore the theory also contains superfluous components.

\section{REFEREN C E S}

1. Velo, G., Zwanziger, D. Phys. Rev., 186, № 5, 1337-1341 (1969).

2. Capri, A. Z. Phys. Rev., 178, № 5, 2427-2433 (1969).

3. Loide, K., Loide, R.-K. Preprint F-6. Tartu, 1977.

4. Amar, V., Dozzio, U. Lett. Nuovo Cim., 12, № 17, 659-662 (1975).

5. Khalil, M. A. K. Progr. Theor. Phys., 58, № 5, 1538-1554 (1977).

6. Cox, W. J. Phys. A: Math. Gen., 15, № 2, 627-635 (1982).

7. Loide, R.-K. ENSV TA Toim., Füüs. Matem., 31, № 4, 451-453 (1982).

8. Kõiv, M., Loide, R.-K., Saar, R. ENSV TA Toim., Füüs. Matem., 31, № 3, 300-303 (1982).

9. Prabhakaran, J., Seetharaman, M., Mathews, P. M. J. Phys. A: Math. Gen., 8, № 4, $560-565$ (1975).

10. Cox, W. J. Phys. A: Math. Gen., 10, № 8, 103-113 (1977).

11. Fisk, C., Tait, W., J. Phys. A: Math. Nucl. Gen., 6, № 3, 383-392 (1973).

12. Khalil, M. A. K., Seetharaman, M. Phys. Rev., D18, № 8, 3040-3044 (1978).

13. Labontè, G. Nuovo Cim., A59, № 3, 263-274 (1980); A65, № 1, 51-63 (1981).

14. Madore, J., Tait, W. Commun. Math. Phys., 30, 201-209 (1973).

15. Ranada, A. F., Sierra, G. Phys. Rev., D22, № 10, 2416-2421 (1980).

16. Aurilia, A., Kobayashi, M., Takahashi, Y. Phys. Rev., D22, № 6, $1368-1374$ (1980).

17. Deser, S., Zumino, B. Phys. Lett., B62, 335-337 (1976).

Tallinn Polytechnic Institute

Received

September 29, 1982

\section{R.-K. LOIDE}

\section{3/2-SPINNI LAINEVORRANDITE MITTEPOHJUSLIKKUSEST}

Kōrgema spinni võrrandite mittepõhjuslikkus minimaalse elektromagnetilise vastasmõju korral on seotud $\beta$-maatriksite nilpotentsusega vaba välja võrrandites. Põhjuslikkust rikkuv levik tuleneb nilpotentsetele alamplokkidele vastavatest omaolekutest.

\section{Р.-К. ЛОИДЕ}

\section{О НЕПРИЧИННОСТИ ВОЛНОВЫХ УРАВНЕНИИ СПИНА $3 / 2$}

Непричинность волновых уравнений высших спинов при минимальном электромагнитном взаимодействии связана с нильпотентностью $\beta$-матриц в уравнениях для свобод"ного поля. Нарушение причинности обусловлено собственными состояниями нильпотентных подблоков. 